\title{
Self-reported physical activity in European adolescents: results from the HELENA (Healthy Lifestyle in Europe by Nutrition in Adolescence) study
}

\author{
Katrien De Cocker ${ }^{1}$ * , Charlene Ottevaere ${ }^{2}$, Michael Sjöström $^{3}$, Luis A Moreno ${ }^{4}$, \\ Julia Wärnberg ${ }^{3,5}$, Jara Valtueña ${ }^{6}$, Yannis Manios7, Sabine Dietrich ${ }^{8}$, Beatrice Mauro 9 , \\ Enrique $G$ Artero $^{10}$, Dénes Molnár ${ }^{11}$, Maria Hagströmer ${ }^{3}$, Jonathan R Ruiz ${ }^{3}$, \\ Katerina Sarri ${ }^{12}$, Anthony Kafatos ${ }^{12}$, Frédéric Gottrand ${ }^{13}$, Stefaan De Henauw ${ }^{2}$, \\ Lea Maes ${ }^{2}$ and Ilse De Bourdeaudhuij ${ }^{1}$ on behalf of the HELENA Study Groupt \\ 'Department of Movement and Sports Sciences, Ghent University, Watersportlaan 2, B-9000 Ghent, Belgium: \\ ${ }^{2}$ Department of Public Health, Ghent University, Ghent, Belgium: ${ }^{3}$ Unit for Preventive Nutrition, Department of \\ Biosciences and Nutrition, Karolinska Institutet, Stockholm, Sweden: ${ }^{4}$ GENUD Research Group, EU Ciencias de \\ la Salud, Universidad de Zaragoza, Zaragoza, Spain: ${ }^{5}$ Department of Nutrition and Metabolism, ICTN, \\ Consejo Superior de Investigaciones Cientificas, Madrid, Spain: ${ }^{6}$ Facultad de Ciencias de la Actvitidad Física y \\ del Delporte - INEF, Universidad Politécnica de Madrid, Madrid, Spain: ${ }^{7}$ Department of Nutrition and Dietetics, \\ Harokopio University, Athens, Greece: ${ }^{8}$ Department of Pediatrics and Adolescent Medicine, Medical University \\ of Vienna, Vienna, Austria: ${ }^{9}$ INRAN National Research Institute on Food and Nutrition, Rome, Italy: \\ ${ }^{10}$ Department of Physiology, School of Medicine, University of Granada, Granada, Spain: "'Department of \\ Paediatrics, University of Pécs, Pécs, Hungary: ${ }^{12}$ Preventive Medicine \& Nutrition Clinic, Department of Social \\ Medicine, Medical School, University of Crete, Heraklion, Crete, Greece: ${ }^{13}$ EA 3925, IFR 114 , Faculty of \\ Medicine, University of Lille 2, Lille, France
}

Submitted 25 August 2009: Accepted 2 February 2010: First published online 18 March 2010

\begin{abstract}
Objective: To describe self-reported physical activity (PA) patterns in the various domains (school, home, transport, leisure time) and intensity categories (walking, moderate PA, vigorous PA) in European adolescents. Furthermore, self-reported PA patterns were evaluated in relation to gender, age category, weight status category and socio-economic status (SES).

Design: Cross-sectional study.

Setting: Ten European cities.

Subjects: In total, 3051 adolescents $(47 \cdot 6 \%$ boys, mean age $14 \cdot 8$ (SD $1 \cdot 2)$ years) completed an adolescent-adapted version of the validated International Physical Activity Questionnaire.

Results: The total sample reported most PA during leisure time ( $485 \mathrm{~min} / \mathrm{week}$ ) and least PA at home $(140 \mathrm{~min} /$ week $)$. Boys reported significantly more school-based PA $(P<0 \cdot 001)$, leisure-time PA $(P=0 \cdot 003)$, vigorous PA $(P<0 \cdot 001)$ and total PA $(P=0 \cdot 002)$ than girls, while girls reported more home-based PA $(P<0 \cdot 001)$ and walking $(P=0 \cdot 002)$ than boys. Self-reported PA at school $(P<0 \cdot 001)$, moderate PA $(P<0 \cdot 001)$, vigorous PA $(P<0 \cdot 001)$ and total PA $(P<0 \cdot 001)$ were significantly higher in younger age groups than in older groups. Groups based on weight status differed significantly only in leisure-time PA $(P=0 \cdot 004)$ and total PA $(P=0 \cdot 003)$, while groups based on SES differed in all PA domains and intensities except transport-related PA and total PA.

Conclusions: The total sample of adolescents reported different scores for the different PA domains and intensity categories. Furthermore, patterns were different according the adolescents' gender, age, weight status and SES.
\end{abstract}

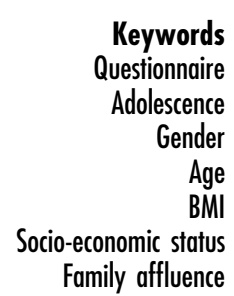

Regular physical activity (PA) during adolescence has short-term (bone and mental health) and long-term (bone

$\uparrow$ See Appendix for full list of HELENA Study Group members. health, breast cancer and sedentary behaviours) health effects $^{(1)}$. Furthermore, there is considerable evidence that the risk factors for chronic diseases are established during this period, influenced by adolescent lifestyle and 
behaviours, in which PA is included ${ }^{(2)}$. Therefore, international health guidelines recommend adolescents to accumulate at least $60 \mathrm{~min}$ of moderate- to vigorousintensity PA daily (60 $\mathrm{min} \mathrm{MVPA} / \mathrm{d})^{(3)}$.

Establishing healthy PA behaviours during adolescence is essential, as PA may track from adolescence to adulthood $^{(1)}$. Also in later life, PA is associated with physiological and psychological health ${ }^{(4)}$. However, a recent worldwide survey in adolescents revealed that only $12-42 \%$ of $13-$ year-olds and $8-37 \%$ of 15 -year-olds met $60 \mathrm{~min}$ MVPA/ $\mathrm{d}^{(5)}$. According to objectively measured data, $4-31 \%$ of European 9- and 15- year-olds accumulated at least $60 \mathrm{~min}$ MVPA $/ \mathrm{d}^{(6)}$. Consequently, the promotion of PA has become an international public health concern. In order to develop PA interventions, it is important to know what the patterns of adolescents' PA are.

Assessing PA in children and adolescents is complicated. Self-report instruments and movement-sensing devices are currently the most frequently used methods for PA assessment in epidemiological research ${ }^{(7)}$. From a public health perspective it is important to get insight into the context, domain or place of PA participation across adolescence. The current health-related PA guidelines recommend youth to be physically active in all domains of living ${ }^{(3)}$. To date, no objective method can distinguish between the different domains of PA. Questionnaires are able to do so; however, previous self-reports focused mainly on one domain, namely leisure-time structured exercise or sport participation. There is a lack of harmonized and comparable data on levels and patterns of the different PA domains in adolescence. Profound investigation of the variations in PA contexts of young people may be essential for the continued study of PA and its impact on health status ${ }^{(8)}$. Furthermore, in order to develop and evaluate PA interventions a better understanding of the level and patterns of adolescents' PA would be useful.

To date, some US ${ }^{(9,10)}$ and Australian ${ }^{(11)}$ large epidemiological studies contain limited information on some domains of adolescent PA. The only study providing European data on adolescent PA behaviour is the Health Behaviour in School-Aged Children Study (HBSC), an international study completed in thirty-four mostly European countries ${ }^{(12)}$. However, the 10- to 16-year-olds were only asked how many days in the past week and in a typical week they were physically active (cumulative activity including sports, school activities, playing with friends and walking to school) for $60 \mathrm{~min}$ or more. No information is available about the separate domains in which adolescents can be active. This is in contrast to the present study, which examined selfreported data in the different domains or contexts of PA behaviour of adolescents in ten European cities, collected during the Healthy Lifestyle in Europe by Nutrition in Adolescence (HELENA) study ${ }^{(13,14)}$.

The aim was to describe patterns in the specific domains (home, school, transport, leisure time) and intensity categories (walking, moderate PA, vigorous PA) of adolescents' self-reported PA. Evaluations were done for the whole
European sample and for groups based on gender, age category $(12.5-13.9$ years, $14.0-14.9$ years, $15 \cdot 0-15.9$ years, $16 \cdot 0-17 \cdot 5$ years), weight status category (underweight, normal weight, overweight, obese) and socio-economic status (SES), namely the educational level of the adolescent's mother and the Family Affluence Scale (FAS).

\section{Methods}

\section{Study design}

The initial motivation behind the HELENA project was to investigate adolescents' nutritional status and lifestyle at European level, using the same methodology throughout several countries ${ }^{(13,14)}$. The basic objective of the project was to obtain standardized, reliable and comparable data from a random sample of European adolescents on a broad battery of relevant nutrition- and health-related parameters ${ }^{(14)}$. The great advantage of the study was the strict standardization of the field work, which precludes to a great extent the kind of immeasurable confounding bias that often interferes when comparing results from isolated studies ${ }^{(15)}$.

Male and female adolescents aged 12.5 to $17 \cdot 49$ years in ten European cities (see Table 1) formed the basis for the sampling selection. The recruitment was done at schools and adolescents from the first two randomly chosen classes of each school were invited to participate. A class was considered eligible if the participation rate was at least $70 \%$. A random cluster sampling of 3000 adolescents stratified for geographical location, age and SES was carried out. In order to ensure true representation of each of the stratified groups, the data were adjusted by a weighting factor to balance the sample according to the theoretical age distribution of the European adolescent population. More details on sampling

Table 1 Sample size by gender, age category and city/country used in the weighted analyses, the HELENA (Healthy Lifestyle in Europe by Nutrition in Adolescence) study

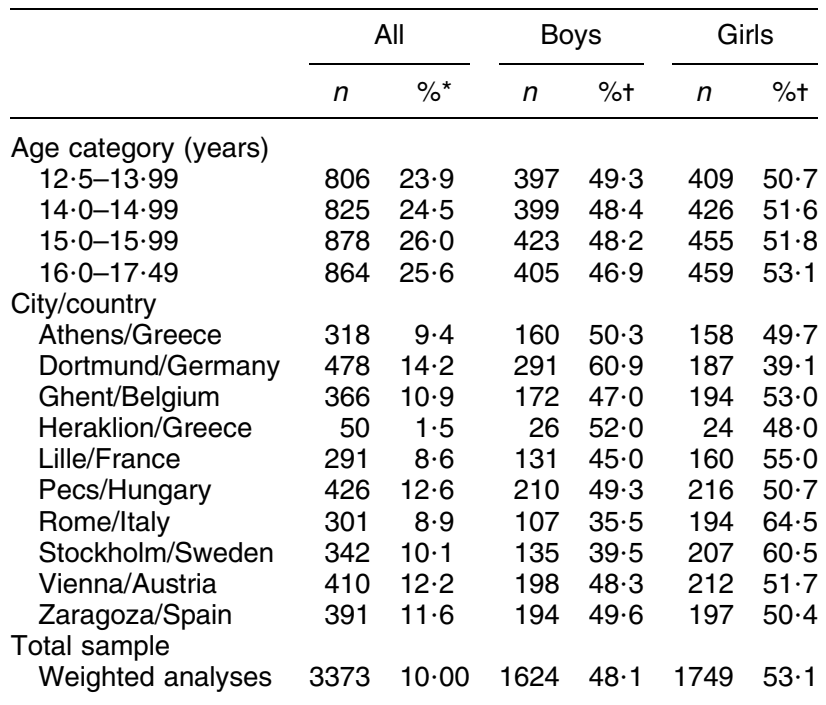

${ }^{*}$ Percentage values expressed in relation to the total sample.

tPercentage values expressed in relation to each age category or city. 
procedures and study design can be found elsewhere ${ }^{(13)}$. The study was approved by the Ethical Committee of each city involved. A signed informed consent form was obtained from both the adolescents and their parents.

\section{Participants and procedure}

In total, 3546 European adolescents received a PA questionnaire (the adolescent-adapted version of the International Physical Activity Questionnaire, IPAQ-A), whereas 3051 (86\%) completed at least $75 \%$ of it (in the HELENA study, participants were included only if they completed at least $75 \%$ of a test ${ }^{(13)}$ ). The questionnaire was handed out to the adolescents during classes and was filled in immediately. The participants were allowed to ask questions to the examiners. The weighted sample size by gender, age category and city can be found in Table 1 .

\section{Measurements}

\section{International Physical Activity Questionnaire for} Adolescents

To assess PA of the last $7 \mathrm{~d}$, an adolescent-adapted version of the International Physical Activity Questionnaire (IPAQ), self-administered and long version, was used. The original questionnaire was developed for adults aged 18-65 years, assessing the different domains of PA (work, transport, house and garden, leisure time). The IPAQ is known to be a valid and reliable instrument to measure PA at population level $^{(16)}$. To adapt the questionnaire to our study population, questions about PA at work were replaced by questions about PA at school, namely physical education, walking, moderate and vigorous PA at school. Furthermore, the items relating to domestic and gardening PA were reduced to one question. A pilot concurrent validation study $^{(17)}$ on this instrument found significant, modest correlations $(r=0 \cdot 17$ to 0.30$)$ between walking, moderate, vigorous and total PA reported in the questionnaire and PA measured by accelerometry for the older adolescents (15-17 years). No significant correlations $(r=-0 \cdot 07$ to $0 \cdot 19)$ could be found for the younger ones (12-14 years). After that study, the IPAQ-A and the data analyses for the accelerometers were adapted. The adjusted IPAQ-A results were compared with the accelerometer data within the HELENA sample. Significant correlations between the IPAQ-A and the Actigraph were found for the whole study sample and when stratified by age and gender $(r=0 \cdot 08$ to $0 \cdot 26)$. While the time spent in moderate PA reported in the IPAQ-A was higher compared with the time measured with the accelerometer, the differences between both instruments were less clear for vigorous PA.

For the IPAQ-A, min/week were computed for PA in the different domains, in total, and for all walking, moderate and vigorous PA based on the guidelines for data processing and analysis of the IPAQ (Guidelines 2005). Furthermore, all scores were truncated at reasonable and realistic levels (see Data analysis), based on previous research ${ }^{(18)}$ and on the frequencies found in the present sample.

\section{Anthropometric measurements}

Adolescents had their height and weight measured by trained researchers in a standardized way ${ }^{(19)}$. The weight was recorded to the nearest $0 \cdot 1 \mathrm{~kg}$, using an electronic scale (type SECA 861). The scale was calibrated and needed no further calibration during the study. The height of the adolescents was recorded to the nearest $0 \cdot 1 \mathrm{~cm}$, using a telescopic height-measuring instrument (type SECA 225). This instrument was calibrated weekly. The measurements were taken after an overnight fast. Light indoor clothing could be worn, excluding shoes, long trousers and sweaters. The BMI of the adolescents was calculated from their measured height and weight $(\mathrm{BMI}=$ weight divided by height squared, $\mathrm{kg} / \mathrm{m}^{2}$ ). International age- and genderspecific cut-off points ${ }^{(20,21)}$ were used to assess BMI category (underweight, normal weight, overweight, obese).

\section{Socio-economic status}

The educational level of adolescent's mother and the FAS were used as indicators of SES.

The educational level of their mother was reported by the adolescents. Health-related aspects are commonly affected by the mother's level of education. Adolescents could indicate whether their mother had a lower education, lower secondary education, higher secondary education or higher education/university degree. This 4-point scale was re-coded into a 2-point scale: low (lower education and lower secondary) and high education (higher secondary and higher/university degree) ${ }^{(22)}$.

The FAS index has shown to be a valid indicator of adolescents' SES and material circumstances ${ }^{(23)}$. Personal and sociodemographic variables included in this index are family car ownership, whether adolescents have their own bedroom, whether Internet is available at home, and the family's computer ownership. The scale ranged from 0 to 8: a score of $0-3$ reflects low familial wealth, 4-5 medium and 6-8 high familial wealth.

\section{Data analysis}

All analyses were conducted using the SPSS for Windows statistical software package version $15 \cdot 0$ (SPSS Inc., Chicago, IL, USA) and adjusted by a weighting factor to ensure true representation of each of the stratified groups. To limit unrealistic high values, the PA scores were truncated in the different domains (school: max $1800 \mathrm{~min} /$ week; home: $\max 1680 \mathrm{~min} /$ week; transport: $\max 1290 \mathrm{~min} /$ week; leisure time: $\max 1680 \mathrm{~min} /$ week; total PA: $\max 2540 \mathrm{~min} /$ week) as well as in the different intensity levels ( $\max 1260 \mathrm{~min} /$ week for walking, moderate PA and vigorous PA, respectively). This was based on previous research with adolescent samples using a comparable questionnaire ${ }^{(18)}$. School-based PA was truncated for thirty-five participants, home-based PA for 
thirty-six, transport-related PA for eighty-seven, leisure-time PA for 153, walking for 363, moderate PA for 328, vigorous PA for fifty-two and total PA for 377 participants. Afterwards, the skewed IPAQ-A data were log-transformed to approximate normal distributions ${ }^{(24,25)}$. Before executing the logtransformation, zero minutes were changed to $0 \cdot 1 \mathrm{~min}$. Parametric analyses were performed on the log-transformed IPAQ-A data; however, the descriptive variables of the nontransformed data (mean, standard deviation, median) are shown in the Results section and tables.

The mean, standard deviation and median of the PA scores in the different domains and the intensity levels are given for the total sample, gender groups, age categories, weight status categories, educational level of the adolescent's mother and FAS categories. To compare the different domain scores (school, home, transport, leisure time) in the total sample, a repeated-measures ANOVA test was conducted. The same test was used to compare the different intensity scores (walking, moderate PA, vigorous PA) mutually in the total sample. Afterwards, for each variable (gender, age, weight status, educational level of the mother and FAS), a multivariate ANOVA was conducted to track differences in the PA scores between groups. When between-subjects tests were significant, additional Tukey post hoc analyses were conducted to examine significant differences. An $\alpha$ level of $P<0.05$ was used to decide upon statistical significance.

\section{Results}

The total sample reported most PA during leisure time (average about $69 \mathrm{~min} / \mathrm{d}$ or $38 \%$ of sum of all domains), less at school $(48 \mathrm{~min} / \mathrm{d}$ or $27 \%)$ and during active transport $(43 \mathrm{~min} / \mathrm{d}$ or $24 \%)$, and the smallest amount of PA $(20 \mathrm{~min} / \mathrm{d}$ or $11 \%)$ was reported at home $(F=735 \cdot 9, P<0 \cdot 001)$. All adolescents reported on average about $65 \mathrm{~min} / \mathrm{d}$ for walking, $70 \mathrm{~min} / \mathrm{d}$ for moderate $\mathrm{PA}$ and $32 \mathrm{~min} / \mathrm{d}$ for vigorous PA. The mean, standard deviation and median ( $\mathrm{min} /$ week) self-reported scores for the different groups based on gender, age, weight status, educational level of the adolescent's mother and FAS are given in Table 2.

Significant gender differences were found for selfreported school-based PA $(P<0 \cdot 001)$, home-based PA $(P<0 \cdot 001)$, leisure-time PA $(P=0 \cdot 003)$, walking $(P=$ $0 \cdot 002)$, vigorous PA $(P<0 \cdot 001)$ and total PA $(P=0 \cdot 002)$. Boys reported significantly more school-based, leisuretime, vigorous and total PA than girls, while girls reported more home-based PA and walking. School-based, moderate, vigorous and total PA also differed significantly (all $P<0 \cdot 001$ ) between the various age categories. Post hoc analyses revealed that the younger groups $(12 \cdot 5-14 \cdot 99$ years) reported more PA than the older ones ( $P$ values all $<0 \cdot 029$ ). Underweight adolescents reported less walking than overweight $(P=0.029)$ and less leisure-time PA than normal weight $(P=0 \cdot 007)$ and overweight $(P=0 \cdot 009)$ adolescents. Furthermore, the total amount of self-reported PA was significantly lower in obese adolescents compared with normal weight $(P=0 \cdot 033)$ and overweight $(P=0 \cdot 010)$ adolescents. All PA scores, except walking, transport-related and total PA, were related to the educational level of the mother. Self-reported school-based $(P<0 \cdot 001)$, leisure-time $(P<0 \cdot 001)$, moderate $(P=0 \cdot 012)$ and vigorous PA $(P<$ $0 \cdot 001)$ showed significant but trivial differences between adolescents whose mother had a low education and those with a high education. In contrast, home-based PA was higher in adolescents whose mother had a low education $(P=0 \cdot 008)$. The FAS was significantly associated only with self-reported walking and vigorous PA. Self-reported walking time was higher among those with low $(P<0 \cdot 001)$ and medium $(P=0 \cdot 001)$ familial wealth than among adolescents with high familial wealth. On the other hand, vigorous PA was reported more among adolescents with medium $(P=0 \cdot 029)$ and high $(P<0 \cdot 001)$ familial wealth (see Table 2).

\section{Discussion}

The present study examined the context and intensity of self-reported PA across European adolescents; also the association with gender, age, weight status and SES was examined. On average, the total sample reported 1158 (SD 776) $\mathrm{min}$ in PA weekly (165 min/d). Most PA was reported during leisure time and least PA was done at home, which was expected as adolescents are not very often responsible for housekeeping and gardening. Furthermore, there was only one question regarding household activities, while the other domains had several questions.

Few, if any, other studies could be found giving information about the domains of adolescent PA. Only one US study ${ }^{(9)}$ had a comparable research question and found that the most frequently reported PA contexts of adolescent exercise and walking were outdoors and at school. No valid comparisons can be made with other European studies conducted in young populations. Mean scores for walking $(500 \mathrm{~min} /$ week, $71 \mathrm{~min} / \mathrm{d})$, moderate PA ( $453 \mathrm{~min} /$ week, $65 \mathrm{~min} / \mathrm{d}$ ) and vigorous PA (227 min/ week, $32 \mathrm{~min} / \mathrm{d}$ ) are relatively high compared with the guideline of $60 \mathrm{~min} \mathrm{MVPA} / \mathrm{d}$. A European study using the IPAQ in adults also showed rather high means for walking (600 min/week), moderate PA (319 min/week) and vigorous PA (282 min/week) ${ }^{(26)}$. Again, no research could be found comparing the relative contribution of walking, moderate and vigorous PA in adolescents' total PA levels. It should be noted here that the high scores could be caused by over-reporting, effected by social desirability or recall biases, a common problem of self-reports. Other important issues that could cause high scores are the failure to recall time in young people and the rounding up of time (e.g. swimming for $1 \mathrm{~h}$ is reported while the adolescent swam only for $30 \mathrm{~min}$ and changed clothes 


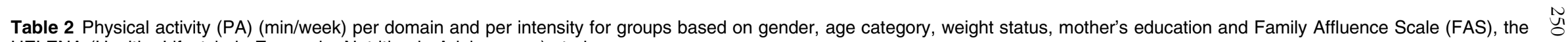
HELENA (Healthy Lifestyle in Europe by Nutrition in Adolescence) study

\begin{tabular}{|c|c|c|c|c|c|c|c|c|c|c|c|}
\hline & & & & \multicolumn{4}{|c|}{ PA domain } & \multicolumn{3}{|c|}{ PA intensity } & \multirow[b]{2}{*}{ Total PA } \\
\hline & & \multicolumn{2}{|l|}{$n$} & School-based PA & Home-based PA & Transport-related PA & Leisure-time PA & Walking & Moderate PA & Vigorous PA & \\
\hline \multirow[t]{6}{*}{ Gender } & Boys & 1624 & Mean & 366 & 126 & 330 & 541 & 450 & 516 & 299 & 1245 \\
\hline & & & & 376 & 213 & 374 & 500 & 420 & 402 & 319 & 800 \\
\hline & & & Median & 240 & 30 & 180 & 380 & 300 & 410 & 190 & 1042 \\
\hline & Girls & 1749 & Mean & 303 & 154 & 277 & 433 & 453 & 468 & 155 & 1077 \\
\hline & & & $\mathrm{SD}$ & 350 & 249 & 324 & 446 & 400 & 384 & 236 & 744 \\
\hline & & & Median & 180 & 60 & 150 & 280 & 320 & 350 & 60 & 860 \\
\hline \multirow[t]{12}{*}{ Age (years) } & $12 \cdot 5-13.99$ & 806 & Mean & 456 & 143 & 303 & 514 & 461 & 590 & 260 & 1296 \\
\hline & & & & 402 & 226 & 344 & 473 & 408 & 391 & 298 & 765 \\
\hline & & & Median & 360 & 60 & 175 & 360 & 335 & 510 & 160 & 1164 \\
\hline & $14 \cdot 0-14.99$ & 825 & Mean & 385 & 140 & 304 & 511 & 465 & 534 & 240 & 1222 \\
\hline & & & $\mathrm{SD}$ & 367 & 240 & 357 & 491 & 421 & 401 & 303 & 790 \\
\hline & & & Median & 270 & 45 & 150 & 360 & 305 & 410 & 120 & 975 \\
\hline & $15 \cdot 0-15 \cdot 99$ & 877 & Mean & 274 & 140 & 301 & 468 & 450 & 442 & 209 & 1095 \\
\hline & & & SD & 321 & 230 & 346 & 462 & 404 & 377 & 270 & 758 \\
\hline & & & Median & 180 & 44 & 160 & 330 & 320 & 330 & 120 & 915 \\
\hline & $16 \cdot 0-17 \cdot 49$ & 864 & Mean & 229 & 139 & 301 & 450 & 434 & 407 & 194 & 1032 \\
\hline & & & & 319 & 236 & 352 & 474 & 405 & 371 & 277 & 764 \\
\hline & & & Median & 124 & 45 & 165 & 283 & 289 & 275 & 70 & 779 \\
\hline \multirow[t]{12}{*}{ Weight status } & Underweight & 203 & Mean & 303 & 133 & 268 & 411 & 398 & 459 & 184 & 1011 \\
\hline & & & SD & 333 & 244 & 323 & 474 & 399 & 376 & 266 & 758 \\
\hline & & & Median & 210 & 30 & 150 & 240 & 225 & 330 & 60 & 749 \\
\hline & Normal & 2427 & Mean & 337 & 142 & 301 & 481 & 445 & 494 & 227 & 1159 \\
\hline & & & SD & 367 & 236 & 349 & 467 & 406 & 393 & 288 & 772 \\
\hline & & & Median & 215 & 45 & 160 & 330 & 300 & 380 & 120 & 950 \\
\hline & Overweight & 567 & Mean & 334 & 143 & 315 & 514 & 492 & 495 & 229 & 1209 \\
\hline & & & $\mathrm{SD}$ & 365 & 231 & 352 & 490 & 418 & 397 & 292 & 776 \\
\hline & & & Median & 220 & 60 & 180 & 360 & 376 & 360 & 120 & 1035 \\
\hline & Obese & 176 & Mean & 311 & 120 & 317 & 531 & 477 & 473 & 219 & 1151 \\
\hline & & & & 345 & 170 & 378 & 534 & 427 & 405 & 290 & 833 \\
\hline & & & Median & 198 & 30 & 172 & 350 & 343 & 343 & 90 & 890 \\
\hline \multirow[t]{6}{*}{ Mother's education } & Low & 1129 & Mean & 326 & 160 & 323 & 487 & 485 & 488 & 217 & 1180 \\
\hline & & & $\mathrm{SD}$ & 391 & 257 & 369 & 495 & 421 & 406 & 291 & 807 \\
\hline & & & Median & 190 & 60 & 175 & 310 & 350 & 360 & 90 & 970 \\
\hline & High & 2077 & Mean & 333 & 127 & 288 & 484 & 430 & 488 & 230 & 1138 \\
\hline & & & SD & 343 & 214 & 335 & 462 & 398 & 384 & 285 & 754 \\
\hline & & & Median & 225 & 45 & 160 & 340 & 290 & 370 & 120 & 930 \\
\hline \multirow[t]{9}{*}{ FAS } & Low & 893 & Mean & 349 & 152 & 333 & 492 & 509 & 491 & 204 & 1206 \\
\hline & & & & 372 & 236 & 375 & 500 & 430 & 395 & 280 & 818 \\
\hline & & & Median & 229 & 60 & 180 & 300 & 377 & 370 & 90 & 996 \\
\hline & Medium & 1406 & Mean & 315 & 135 & 294 & 477 & 443 & 475 & 223 & 1124 \\
\hline & & & $\mathrm{SD}$ & 353 & 235 & 335 & 469 & 398 & 385 & 289 & 757 \\
\hline & & & Median & 200 & 40 & 175 & 337 & 300 & 360 & 120 & 930 \\
\hline & High & 1053 & Mean & 344 & 136 & 286 & 490 & 415 & 511 & 245 & 1160 \\
\hline & & & & 371 & 224 & 344 & 460 & 402 & 401 & 290 & 762 \\
\hline & & & Median & 220 & 45 & 150 & 341 & 270 & 390 & 148 & 960 \\
\hline
\end{tabular}


$2 \times 15 \mathrm{~min})^{(27)}$. Despite these limitations, a survey like this is valuable to determine the domain or context of PA in adolescents.

The present results on context and intensity of PA reveal that already in adolescents walking and moderate-intensity PA contribute more to total PA than vigorous PA, and that adolescents get most of their total minutes of activity during their leisure time (about 38\%) and school (about 27\%). This also shows that schools play a major role in contributing to adequate activity levels in adolescents (about 5-6 h/week) throughout Europe, and pleads for keeping the compulsory physical education lessons in most European countries and the initiatives developed to include PA in breaks, recess and after-school programmes.

As found in other studies using self-reports ${ }^{(5,11,28)}$ or objective PA data ${ }^{(29-31)}$, boys in the present study were observed to be more active than girls. Similar results were found earlier in Europe ${ }^{(27,32)}$. Despite the fact that boys reported a higher amount of total PA than girls, the girls in the present study reported more PA at home and more walking. Perhaps this can be explained by social structures: most of the time, adult women are more responsible for the housekeeping and do more walking activities (e.g. shopping) than men ${ }^{(33)}$. This is probably also true among a young population, as found too by Olds et $a l^{(11)}$.

Furthermore, our results showed that older adolescents reported less total PA than younger ones. The difference in total self-reported PA between the age categories is mostly due to school-based PA: school-based PA clearly differed between the various age categories, while PA in the other domains did not. Olds et al. ${ }^{(11)}$ also found that free play (at school and outside school) decreased across ages in Australia. In development and evaluation of PA interventions, attention should be given to this drop in school-based PA across secondary school. The age-related decline in PA was also found in other cohort studies ${ }^{(5,27,29)}$ and in longitudinal studies ${ }^{(34)}$. However, these studies often presume that especially leisure-time activities drop, while the present results suggest that the main decrease in PA with age could be related to school-based PA.

In contrast to other studies showing differences in PA between normal weight and overweight adolescents ${ }^{(18,35)}$, less clear differences were found in the present adolescents. In agreement with our findings, some other studies could not confirm a negative association between PA and body fatness ${ }^{(36,37)}$ or found that underweight adolescents were less likely to be physically active ${ }^{(10)}$.

A final variable that significantly affected adolescents' selfreported PA was SES. First, the educational level of the mother affected some PA scores, like found by Hanson and Chen ${ }^{(38)}$. A second SES indicator, the FAS, showed a negative association with walking and a positive association with vigorous PA. Also a Chinese ${ }^{(39)}$ and an Australian ${ }^{(40)}$ study found associations between family SES and walking.

The present study had some limitations that need to be considered. First, few validation studies were executed with the IPAQ-A, showing low to modest correlations between PA reported in the questionnaire and PA levels based on accelerometer data ${ }^{(17)}$. Other studies also found weak to moderate but significant associations between questionnaires and accelerometer data in adolescents ${ }^{(41,42)}$. Second, due to the cross-sectional nature of the study, no causal conclusions can be drawn. Also the results on age and PA do not reflect longitudinal trends. Although selfreported data provide information on the intensity and context of PA, as stated before, self-reported measures can be over-reported. Strengths of the present study include the large sample size, the inclusion of different countries and information on PA context and level of intensity.

To conclude, the present study provided data on the domain and intensity category of European adolescents' self-reported PA. Differences in PA domain and intensity were found according to gender, age, weight status and SES of the adolescents. These data may provide interesting information for the development of PA interventions in adolescence. Moreover, the data may serve as baseline to evaluate the effects of PA promotion programmes, but further comparisons with objective data from the same individuals may be appropriate.

\section{Acknowledgements}

Sources of funding: The HELENA Study took place with the financial support of the European Community Sixth RTD Framework Programme (Contract FOOD-CT: 2005007034) and the ALPHA-project in the framework of the Public Health Programme (2006120). The content of this paper reflects the authors' views only and the European Community is not liable for any use that may be made of the information contained herein. Conflict of interest declaration: There are no perceived conflicts of interest related to the research reported in the manuscript. All authors agree with the content of the manuscript and no author has interests that might be interpreted as influencing the research. Authors' contributions: K.D.C. contributed to the conception and design, analysed the data, led the writing of the paper and wrote the manuscript, while C.O. and I.D.B. participated in designing the statistical analyses and interpretation of the data. C.O., M.S., L.A.M., J.W., J.V., Y.M., S.D., B.M., E.G.A., D.M., M.H., J.R.R., K.S., A.K., F.G., S.D.H., L.M. and I.D.B. contributed to the study conception and design, and provided feedback on the manuscript. Acknowledgements: We gratefully acknowledge all participating adolescents and their parents and teachers for their collaboration, and all members of the HELENA Study Group involved in the field work.

\section{References}

1. Hallal PC, Victora CG, Azevedo MR et al. (2006) Adolescent physical activity and health: a systematic review. Sports Med 36, 1019-1030. 
2. Summerbell CD, Waters E, Edmunds LD et al. (2005) Interventions for preventing obesity in children. Cochrane Database Syst Rev 20, issue 3, CD001871.

3. US Department of Health and Human Services, Physical Activity Guidelines Advisory Committee (2008) 2008 Physical Activity Guidelines for Americans. http:// www.health.gov/paguidelines/pdf/paguide.pdf (accessed April 2009).

4. US Department of Health and Human Services (1996) Physical Activity and Health: A Report of the Surgeon General. Atlanta, GA: US Department of Health and Human Services, Centers for Disease Control and Prevention.

5. Currie C, Gabhainn S, Godeau E et al. (editors) (2008) Inequalities in Young People's Health: HBSC International Report from the 2005/2006 Survey. Copenhagen: WHO Regional Office for Europe.

6. Nilsson A, Anderssen SA, Andersen LB et al. (2009) Between- and within-day variability in physical activity and inactivity in 9- and 15-year-old European children. Scand J Med Sci Sports 19, 10-18.

7. Corder K, Ekelund U, Steele RM et al. (2008) Assessment of physical activity in youth. J Appl Physiol 105, 977-987.

8. Kristensen PL, Korsholm L, Moller NC et al. (2008) Sources of variation in habitual physical activity of children and adolescents: the European Youth Heart Study. Scand J Med Sci Sports 18, 298-308.

9. Dunton G, Whalen C, Jamner L et al. (2007) Mapping the social and physical contexts of physical activity across adolescence using ecological momentary assessment. Ann Behav Med 34, 144-153.

10. Levin S, Lowry R, Brown DR et al. (2003) Physical activity and body mass index among US adolescents: Youth Risk Behavior Survey, 1999. Arch Pediatr Adolesc Med 157, 816-820.

11. Olds T, Wake M, Patton G et al. (2009) How do school-day activity patterns differ with age and gender across adolescence? J Adolesc Health 44, 64-72.

12. Janssen I, Katzmarzyk P, Boyce W et al. (2005) Comparison of overweight and obesity prevalence in school-aged youth from 34 countries and their relationships with physical activity and dietary patterns. Obes Rev 6, 123-132.

13. Moreno LA, De Henauw S, González-Gross M et al. (2008) Design and implementation of the Healthy Lifestyle in Europe by Nutrition in Adolescence Cross-Sectional Study. Int J Obes (Lond) 32, Suppl. 5, S4-S11.

14. De Henauw S, Gottrand F, De Bourdeaudhuij I et al. on behalf of the HELENA Study Group (2007) Nutritional status and lifestyles of adolescents in a public health perspective. The HELENA Project - Healthy Lifestyle in Europe by Nutrition in Adolescence. J Public Health 15, 187-197.

15. Moreno LA, González-Gross M, Kersting M et al. on behalf of the HELENA Study Group (2008) Assessing, understanding and modifying nutritional status, eating habits and physical activity in European adolescents: The HELENA (Healthy Lifestyle in Europe by Nutrition in Adolescence) Study. Public Health Nutr 11, 288-299.

16. Craig C, Marshall AL, Sjöström M et al. (2003) International Physical Activity Questionnaire: 12-country reliability and validity. Med Sci Sports Exerc 35, 1381-1395.

17. Hagströmer M, Bergman P, De Bourdeaudhuij I et al. on behalf of the HELENA Study Group (2008) Concurrent validity of a modified version of the International Physical Activity Questionnaire (IPAQ-A) in European adolescents The HELENA study. Int J Obes (Lond) 32, Suppl. 5, S42-S48.

18. Haerens L, Deforche B, Maes L et al. (2007) Physical activity and endurance in normal weight versus overweight boys and girls. $J$ Sports Med Phys Fitness 47, 344-350.
19. Nagy E, Vicente-Rodriguez G, Manios Y et al. on behalf of the HELENA Study Group (2008) Harmonization process and reliability assessment of anthropometric measurements in a multicenter epidemiologic study in adolescents. Int $J$ Obes (Lond) 32, Suppl. 5, S58-S65.

20. Cole TJ, Bellizzi MC, Flegal KM et al. (2000) Establishing a standard definition for child overweight and obesity worldwide: international survey. BMJ 320, 1240-1243.

21. Cole TJ, Flegal KM, Nicholls D et al. (2007) Body mass index cut offs to define thinness in children and adolescents: international survey. BMJ 335, 194.

22. Von Rueden U, Gosch A, Rajmil L et al., the European KIDSCREEN Group (2006) Socioeconomic determinants of health related quality of life in childhood and adolescence: results from a European study. I Epidemiol Community Health 60, 130-135.

23. Currie C, Molcho M, Boyce W et al. (2008) Researching health inequalities in adolescents: the development of the Health Behaviour in School-Aged Children (HBSC) Family Affluence Scale. Soc Sci Med 66, 1429-1436.

24. Napolitano M, Fotheringham M, Tate D et al. (2003) Evaluation of an internet-based physical activity intervention: a preliminary investigation. Ann Behav Med 25, 92-99.

25. Rzewnicki R, Auweele Y \& De Bourdeaudhuij I (2002) Addressing overreporting on the International Physical Activity Questionnaire (IPAQ) telephone survey with a population sample. Public Health Nutr 6, 299-305.

26. Rütten A, Ziemainz H, Schena F et al. (2003) Using different physical activity measurements in eight European countries. Results of the European Physical Activity Surveillance System (EUPASS) time series survey. Public Health Nutr $\mathbf{6}$, $371-376$

27. Armstrong N \& Welsman JR (2006) The physical activity patterns of European youth with reference to methods of assessment. Sports Med 36, 1067-1086.

28. Tammelin T, Ekelund U, Remes J et al. (2007) Physical activity and sedentary behaviors among Finnish youth. Med Sci Sports Exerc 39, 1067-1074.

29. Trost S, Pate R, Sallis J et al. (2002) Age and gender differences in objectively measured physical activity in youth. Med Sci Sports Exerc 34, 350-355.

30. Metcalf B, Voss L, Hosking J et al. (2008) Physical activity at the government recommended level and obesity-related health outcomes: a longitudinal study (Early Bird 37). Arch Dis Child 93, 772-777.

31. Pate R, Freedson P, Sallis J et al. (2002) Compliance with physical activity guidelines: prevalence in a population of children and youth. Ann Epidemiol 12, 303-308.

32. Pahkala K, Heinonen OJ, Lagström H et al. (2007) Leisuretime physical activity of 13 year-old adolescents. Scand J Med Sci Sports 17, 324-330.

33. Sayer L (2005) Gender, time and inequality: trends in women's and men's paid work, unpaid work and free time. Soc Forces 84, 285-301.

34. Nelson M, Neumark-Stzainer D, Hannan PJ et al. (2006) Longitudinal and secular trends in physical activity and sedentary behaviour during adolescence. Pediatrics 118, 1627-1634.

35. Steinbeck K (2001) The importance of physical activity in the prevention of overweight and obesity in childhood: a review and an opinion. Obes Rev 2, 117-130.

36. Ekelund U, Poortvliet E, Nilsson A et al. (2001) Physical activity in relation to aerobic fitness and body fat in 14- to 15-year-old boys and girls. Eur J Appl Physiol 85, 195-201.

37. Katzmarzyk PT, Malina RM, Song TM et al. (1998) Physical activity and health-related fitness in youth: a multivariate analysis. Med Sci Sports Exerc 30, 709-714. 
38. Hanson M \& Chen E (2007) Socioeconomic status and health behaviors in adolescence: a review of the literature. $J$ Behav Med 30, 263-285.

39. Shi Z, Lien N, Kumar B et al. (2006) Physical activity and associated socio-demographic factors among school adolescents in Jiangsu Province, China. Prev Med 43, 218-221.

40. Giles-Corti B \& Donovan R (2002) Socioeconomic status differences in recreational physical activity levels and real

\section{Appendix}

\section{The HELENA Study Group}

Co-ordinator: Luis A. Moreno.

Core Group members: Luis A. Moreno, Frédéric Gottrand, Stefaan De Henauw, Marcela González-Gross, Chantal Gilbert.

Steering Committee: Anthony Kafatos (President), Luis A. Moreno, Christian Libersa, Stefaan De Henauw, Jackie Sánchez, Frédéric Gottrand, Mathilde Kersting, Michael Sjöstrom, Dénes Molnár, Marcela González-Gross, Jean Dallongeville, Chantal Gilbert, Gunnar Hall, Lea Maes, Luca Scalfi.

Project Manager: Pilar Meléndez.

Universidad de Zaragoza (Spain): Luis A. Moreno, Jesús Fleta, José A. Casajús, Gerardo Rodríguez, Concepción Tomás, María I. Mesana, Germán Vicente-Rodríguez, Adoración Villarroya, Carlos M. Gil, Ignacio Ara, Juan Revenga, Carmen Lachen, Juan Fernández Alvira, Gloria Bueno, Aurora Lázaro, Olga Bueno, Juan F. León, Jesús Ma Garagorri, Manuel Bueno, Juan Pablo Rey López, Iris Iglesia, Paula Velasco, Silvia Bel.

Consejo Superior de Investigaciones Científicas (Spain): Ascensión Marcos, Julia Wärnberg, Esther Nova, Sonia Gómez, Esperanza Ligia Díaz, Javier Romeo, Ana Veses, Mari Angeles Puertollano, Belén Zapatera, Tamara Pozo.

Université de Lille 2 (France): Laurent Beghin, Christian Libersa, Frédéric Gottrand, Catalina Iliescu, Juliana Von Berlepsch.

Research Institute of Child Nutrition Dortmund, Rheinische Friedrich-Wilhelms-Universität Bonn (Germany): Mathilde Kersting, Wolfgang Sichert-Hellert, Ellen Koeppen.

Pécsi Tudományegyetem (University of Pécs) (Hungary): Dénes Molnar, Eva Erhardt, Katalin Csernus, Katalin Török, Szilvia Bokor, Mrs. Angster, Enikö Nagy, Orsolya Kovács, Judit Répasi.

University of Crete School of Medicine (Greece): Anthony Kafatos, Caroline Codrington, María Plada, Angeliki Papadaki, Katerina Sarri, Anna Viskadourou, Christos Hatzis, Michael Kiriakakis, George Tsibinos, Constantine Vardavas Manolis Sbokos, Eva Protoyeraki, Maria Fasoulaki.

Institut für Ernährungs- und Lebensmittelwissenschaften - Ernährungphysiologie, Rheinische Friedrich-WilhelmsUniversität (Germany): Peter Stehle, Klaus Pietrzik, and perceived access to a supportive physical environment. Prev Med 35, 601-611.

41. McMurray R, Ring K, Treuth M et al. (2004) Comparison of two approaches to structured physical activity surveys for adolescents. Med Sci Sports Exerc 36, 2135-2143.

42. Wong S, Leatherdale S \& Manske S (2006) Reliability and validity of a school-based physical activity questionnaire. Med Sci Sports Exerc 38, 1593-1600.
Marcela González-Gross, Christina Breidenassel, Andre Spinneker, Jasmin Al-Tahan, Miriam Segoviano, Anke Berchtold, Christine Bierschbach, Erika Blatzheim, Adelheid Schuch, Petra Pickert.

University of Granada (Spain): Manuel J. Castillo Garzón, Ángel Gutiérrez Sáinz, Francisco B. Ortega Porcel, Jonatan Ruiz Ruiz, Enrique García Artero, Vanesa España Romero, David Jiménez Pavón, Cristóbal Sánchez Muñoz, Victor Soto, Palma Chillón, Jose M. Heredia, Virginia Aparicio, Pedro Baena, Claudia M. Cardia, Ana Carbonell.

Istituto Nazionalen di Ricerca per gli Alimenti e la Nutrizione (Italy): Davide Arcella, Elena Azzini, Emma Barrison, Noemi Bevilacqua, Pasquale Buonocore, Giovina Catasta, Laura Censi, Donatella Ciarapica, Paola D'Acapito, Marika Ferrari, Myriam Galfo, Cinzia Le Donne, Catherine Leclercq, Giuseppe Maiani, Beatrice Mauro, Lorenza Mistura, Antonella Pasquali, Raffaela Piccinelli, Angela Polito, Raffaella Spada, Stefania Sette, Maria Zaccaria.

University of Napoli 'Federico II' Department of Food Science (Italy): Luca Scalfi, Paola Vitaglione, Concetta Montagnese.

Ghent University (Belgium): Ilse De Bourdeaudhuij, Stefaan De Henauw, Tineke De Vriendt, Lea Maes, Christophe Matthys, Carine Vereecken, Mieke de Maeyer, Charlene Ottevaere.

Medical University of Vienna (Austria): Kurt Widhalm, Katharina Phillipp, Sabine Dietrich, Birgit Kubelka, Marion Boriss-Riedl.

Harokopio University (Greece): Yannis Manios, Eva Grammatikaki, Zoi Bouloubasi, Tina Louisa Cook, Sofia Eleutheriou, Orsalia Consta, George Moschonis, Ioanna Katsaroli, George Kraniou, Stalo Papoutsou, Despoina Keke, Ioanna Petraki, Elena Bellou, Sofia Tanagra, Kostalenia Kallianoti, Dionysia Argyropoulou, Katerina Kondaki, Stamatoula Tsikrika, Christos Karaiskos.

Institut Pasteur de Lille (France): Jean Dallongeville, Aline Meirhaeghe.

Karolinska Institutet (Sweden): Michael Sjöstrom, Patrick Bergman, María Hagströmer, Lena Hallström, Mårten Hallberg, Eric Poortvliet, Julia Wärnberg, Nico Rizzo, Linda Beckman, Anita Hurtig Wennlöf, Emma Patterson, Lydia Kwak, Lars Cernerud, Per Tillgren, Stefaan Sörensen.

Asociación de Investigación de la Industria Agroalimentaria (Spain): Jackie Sánchez-Molero, Elena Picó, Maite Navarro, Blanca Viadel, José Enrique Carreres, Gema Merino, Rosa Sanjuán, María Lorente, María José Sánchez, Sara Castelló. 
Campden \& Chorleywood Food Research Association (UK): Chantal Gilbert, Sarah Thomas, Peter Burguess.

SIK - Institutet foer Livsmedel och Bioteknik (Sweden): Gunnar Hall, Annika Astrom, Anna Sverkén, Agneta Broberg.

Meurice Recherche \& Development asbl (Belgium): Annick Masson, Claire Lehoux, Pascal Brabant, Philippe Pate, Laurence Fontaine.

Campden \& Chorleywood Food Development Institute (Hungary): Andras Sebok, Tunde Kuti, Adrienn Hegyi.

Productos Aditivos SA (Spain): Cristina Maldonado, Ana Llorente.
Cárnicas Serrano SL (Spain): Emilio García.

Cederroth International AB (Sweden): Holger von Fircks, Marianne Lilja Hallberg, Maria Messerer.

Lantmännen Food RED (Sweden): Mats Larsson, Helena Fredriksson, Viola Adamsson, Ingmar Börjesson.

European Food Information Council (Belgium): Laura Fernández, Laura Smillie, Josephine Wills.

Universidad Politécnica de Madrid (Spain): Marcela González-Gross, Agustín Meléndez, Pedro J. Benito, Javier Calderón, David Jiménez-Pavón, Jara Valtueña, Paloma Navarro, Alejandro Urzanqui, Ulrike Albers, Raquel Pedrero, Juan José Gómez Lorente. 